\title{
Reversal of forebrain
}

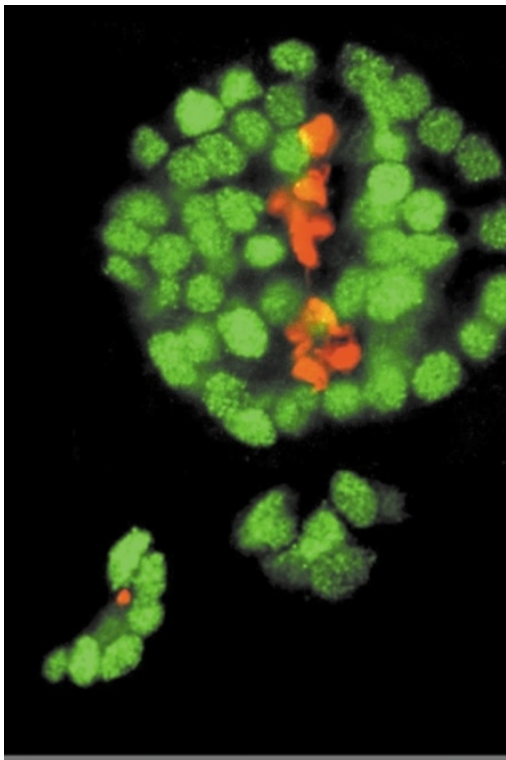

Diencephalon of a 3.5-day-old Zebrafish embryo. Courtesy of College London, London, UK. Stephen Wilson, University

Functional asymmetries of the vertebrate brain, such as the localization of speech areas to the left hemisphere in humans, have been well documented. Indeed, although vertebrate brains are largely symmetrical, neuroanatomical and cognitive left-right differences have been identified in all species that have been examined. However, it has remained uncertain how these asymmetries arise during development. Evidence for genetic control of forebrain asymmetry is now emerging from studies on the zebrafish brain, which has several features that show overt lateral asymmetry. Research has focused on two diencephalic structures; the habenular nucleus, which is larger on the left side than the right, and the parapineal organ, which is located to the left of the midline.

Concha et al. describe the effects of disrupting Nodal signalling on the development of these two structures. The Nodal pathway is conserved between species and is important for the generation of left-right asymmetry in the heart and viscera. The nodal homologue cyclops $(c y c)$ and the homeobox gene pitx 2 are normally expressed only on the left side of the developing diencephalon. The authors obtained evidence that early activity of the Cyclops cofactor, one-eyed pinhead (Oep), leads to repression of cyc and pitx 2 on both sides of the diencephalon, whereas later Oep-dependent signalling, combined with activity of the transcription factor gene Schmalspur (Sur), overcomes this repression on the left side. They showed that embryos mutant for sur or lacking late Oep function did not express cyc and pitx 2 in the diencephalon. Conversely, ablation of the midline results in bilateral expression of these genes, indicating that signals from this tissue are required for their repression. In both cases, the diencephalon still developed asymmetrically, but the laterality of the asymmetry became random.

So, Nodal signalling does not seem to be essential for asymmetric brain development per se, but it determines the direction of the asymmetry. Further experiments will elucidate the mechanisms that initiate the development of brain asymmetry, and show whether anatomical and functional asymmetries are influenced by common factors.

Heather Wood

\section{(1) References and links}

ORIGINAL RESEARCH PAPER Concha, M. L. et al. A Nodal signalling pathway regulates the laterality of neuroanatomical asymmetries in the zebrafish forebrain. Neuron $\mathbf{2 8}$, 399-409 (2000)

FURTHER READING Burdine, R. D. \& Schier, A. F. Conserved and divergent mechanisms in left-right axis development. Genes Dev. 14, 763-776 (2000)

WEB SITE UCL Zebrafish Group

ENCYCLOPEDIA OF LIFE SCIENCE Zebrafish embryo as a developmental system

STEM CELLS

\section{Crossing blood-brain barriers}

Recent advances in cloning and embryonic stem cell technology have raised hopes that tissue for transplantation may be grown in vitro from a single somatic cell taken from the recipient. This would solve the problems of organ shortage and transplant rejection in one fell swoop. However, in reality, embryo cloning is extremely inefficient, not to mention controversial, and human stem-cell lines have proved more difficult to establish and maintain than their mouse counterparts. Therefore, it might be argued that time would be better spent looking for alternative, more accessible, sources of pluripotent stem cells. It is becoming increasingly clear that lineage-specific stem cells, and even fully differentiated cells, show remarkable capacities for switching fate. Now, Science reports on two independent studies showing that bone-marrowderived cells can colonize the brain and differentiate into neurons.

Brazelton et al. used adult bone marrow cells expressing green fluorescent protein (GFP) to rescue lethally irradiated adult mice. By cell sorting, they found that up to $20 \%$ of GFPpositive cells in the brain lacked expression of haematopoietic markers. However, this did not prove that they were neurons, as bone marrow cells can also generate microglia. To overcome this problem, the researchers used laser scanning confocal microscopy to look for expression of GFP and neuronal markers together in olfactory bulb (OB) sections. 8-12 weeks after transplantation, $0.2-0.3 \%$ of the total $\mathrm{OB}$ neuronal population was GFP-positive. Most of these cells were found in the superficial axon and

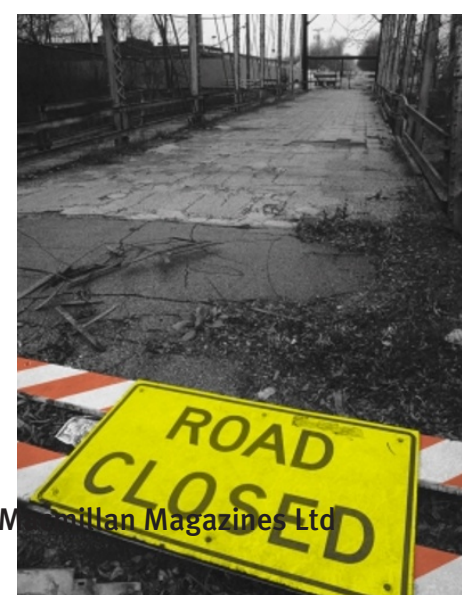

glomerular layers, and most resembled neuroblasts rather than mature neurons. This work shows that profound changes in cell fate occur in marrowderived cells located in the CNS.

Mezey et al. used mice mutant for the PU.1 gene, which are unable to produce cells of the lymphoid and myeloid lineages. These mice die unless they receive a bone-marrow transplant within 48 hours of birth. Female mutant mice received an intraperitoneal injection of bone marrow from an adult wild-type male donor during the first postnatal day. Their brains were examined at 1-4 months of age to look for expression of the neuronal marker NeuN, combined with the presence of the $\mathrm{Y}$ chromosome. They found that $0.3-2.3 \%$ of NeuN-positive cells were of male origin. The double-labelled cells were found predominantly, but not exclusively, in the cerebral cortex.

Bone-marrow cells have already been shown to have neurogenic potential in vitro, but these new findings are important because they show that these cells can also differentiate into neurons in vivo. However, predictions about possible therapeutic applications are probably premature. For instance, the recipient mice used in these studies were either newborn, or had received doses of radiation that could mildly injure the brain tissue, perhaps creating a more permissive environment for neuronal differentiation. To take full advantage of the possibilities presented by these experiments, it might be necessary to develop techniques for isolating the subset of cells that show neurogenic potential, or to identify growth factors that encourage bone marrow cells to differentiate as neurons. What we can conclude is that these data lend a new meaning to the phrase 'crossing the blood-brain barrier'.

(2) References and links Heather Wood ORIGINAL RESEARCH PAPERS Brazelton, T. R. et al. From marrow to brain: expression of neuronal phenotypes in adult mice. Science 290, 1775-1779 (2000) | Mezey, E. et al. Turning blood into brain: cells bearing neuronal antigens generated in vivo from bone marrow. Science 290, 1779-1782 (2000) WEB SITE Helen Blau's laboratory 